\title{
Research on the Role of Art Creation in Ceramic Material Product Design Based on the Perspective of Innovation
}

\author{
He yueming ${ }^{1}$ \\ ${ }^{1}$ Fuzhou University (Xiamen Academy of Arts and Design, Xiamen, 361021)
}

\begin{abstract}
Materials are the material basis for product design, and many products design needs to be achieved through Ceramic materials. Materials are not only the material basis of products, but also the hallmark of social civilization. The choice of Ceramic materials is not only one of the decisive factors for the success of product design, but also the key to the artistic aesthetic of the product. The perfect shape, color and function are important, and the material texture can not be ignored, it will affect the final effect of product design. The application of Ceramic materials in product design is too stylized, which seriously restricts the innovative role of Ceramic materials in product design. It is necessary to re-recognize the design characteristics and functions of Ceramic materials and explore their application methods and means. This paper analyzes the importance and complexity of material selection in product design under the perspective of innovation, and further explores the role of material art creation in product design, summarizes the basic principles and methods of material selection in product design, and hopes to pass scientific Ceramic materials. Choose to optimize the design of your product.
\end{abstract}

\section{Introduction}

Ceramic materials are the main expression methods and means used by product designers and artists to express people's feelings about life in different periods, cultures and regions. They are also the most important carriers for constructing social and cultural forms [1]. The texture of the material is visual and tactile, as well as psychological association and symbolic meaning through the surface features of the product [2]. The perfect shape, color and function are important, and the material texture can not be ignored, it will affect the final effect of product design [3]. Humans constantly discover and invent new Ceramic materials in their creation activities and use them to create everything around us. In product design, material is the material condition of product design, which is the basis and premise of product design. The material affects product design with its own characteristics [4]. The design material is a substance that constitutes the product shape and does not depend on human consciousness and exists objectively. It constitutes the basis of product design [5]. Designers who combine the various design concepts with their characteristics after mastering the functional properties, sensory characteristics and symbolic semantics of Ceramic materials can give new ideas to product design [6]. Designers must fully understand the material properties, processing technology, environment, standards and other relevant factors, master the principles and methods of material selection, through scientific analysis and thinking, in order to better select the appropriate Ceramic materials for the design of the product [7].
In modern society, Ceramic materials are not only the material basis of products, but also the symbol of social civilization. Choosing the right Ceramic materials and making rational use is also a prerequisite for the formation of excellent products [8]. In the product design process, the choice of Ceramic materials is an essential part of the process, and sometimes even the primary link [9]. It affects the structural design, manufacturing, cost, performance, life and product quality and even the production cycle [10]. Material is the material basis of product composition and a symbol of social civilization. Choosing the right material is a prerequisite for excellent product design [11]. The texture semantics of Ceramic materials is the information transfer of product material properties, texture and texture, which is closely related to product design [12]. On the one hand, the material becomes the material basis of the creation and the basic content of the constituent items, on the other hand, it becomes the intermediary and object for people to realize their own purposes and ideals, and forms a close relationship with people [13]. The application of Ceramic materials in product design is too stylized, which seriously restricts the innovative role of Ceramic materials in product design. It is necessary to re-recognize the design characteristics and functions of Ceramic materials and explore their application methods and means [14]. Only by correctly using the communication function of the material texture semantics can the product be accurately designed and the product can better serve the public's life [15].

Material is the material basis of product design, and any product design must be realized through Ceramic 
materials, otherwise it can only stay in the concept stage. The design material conveys different information and judgments to us with its own inherent characteristics and texture characteristics, which directly affects the success or failure of product design [16]. Every advancement in material innovation or application will lead to a radical change in the field of product design, which will lead to the birth of a new generation of products and realize people's dreams one after another [17]. The choice of Ceramic materials is not only one of the decisive factors for the success of product design, but also the key to the artistic aesthetic of the product. In addition to its functional properties, Ceramic materials also have their own unique sensory characteristics, which imply emotional information corresponding to people's hearts [18]. Due to the emergence of new Ceramic materials and new processes, the selection of Ceramic materials for product design has been expanding, and new Ceramic materials have become the source of product art design innovation [19]. In modern product design, it is necessary to comprehensively analyze the function and modeling elements of the product, and at the same time, to understand the aesthetic taste of the consumer, and use the systemic thinking method to guide the choice of Ceramic materials [20]. This paper explores the relationship between product design and Ceramic materials, analyzes the role of material art creation in product design under the perspective of innovation, summarizes the basic principles and methods of material selection in product design, and hopes to optimize product design through scientific material selection.

\section{Material Art Creation in Product Design}

\subsection{Design Characteristics and Functions of Ceramic materials}

Excellent products are often a harmonious unity of functions, forms, Ceramic materials, processes and other elements. As the basic carrier of product substances and mental functions, Ceramic materials often have a decisive influence on the design results. People's clothing, food, shelter, and transportation are inseparable from products, and they are inseparable from all kinds of Ceramic materials. Ceramic materials must have the right properties to meet the functional and economic availability of human survival and development [21]. Under the premise of ensuring satisfactory performance of Ceramic materials, it is an important aspect of material science researchers to use various measures to reduce the environmental impact of Ceramic materials during processing and use. After deliberately processed Ceramic materials, the surface effect is rich, but the value of the design does not necessarily increase. In many cases, the imposed colors and textures appear to be artificial and have the opposite effect.

The microscopic morphology of the material is a major factor affecting the dielectric properties of the microwave dielectric material. The grain distribution, grain size, grain shape, density, pore size, and the like of the sample were directly observed by electron microscopic analysis.
Generally, for microwave dielectric Ceramic materials, the larger the grain size, the less the number of grain boundaries is, and the smaller the dielectric loss is. Fig. 1 shows a ceramic substrate of a microwave medium.

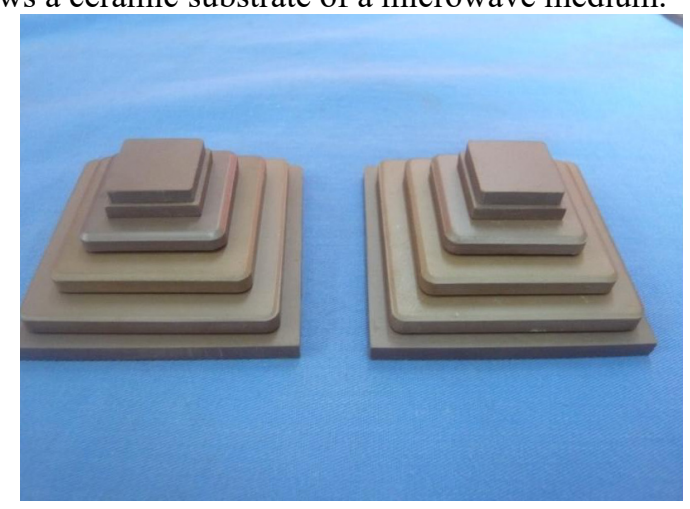

Fig. 1 Microwave dielectric ceramic substrate

We usually appreciate the design of a particular item from the usual form and function of an item, but often overlook the study of the particular material that forms the item or function. Functional factors mainly include practical functions, aesthetic functions and safety performance. The practical function is the basic function of the product and is the dominant factor in the selection of Ceramic materials in the design. Ceramic materials need to be processed by technology to become the product that people want, and the achievability of technology will directly affect the designer's choice of Ceramic materials [22]. The formation of new material processes has also had an impact on its material. Such as stamping, stretching and other processes, but also bring a lot of changes, so that the morphological texture is diversified. In different historical periods, due to changes in social economy, culture, technology and other conditions, product design has also focused on the application of material properties.

\subsection{Application of Material Design Characteristics}

The stylized application method stems from people's cognition of stale and rigid Ceramic materials, so it is necessary to eliminate stereotypes and re-recognize the design characteristics and functions of Ceramic materials. Designers should be proficient in the cooperation between Ceramic materials, processing technology and form, and use various Ceramic materials reasonably and effectively, and design new products based on economic, practical and aesthetic needs, so that Ceramic materials can be processed into people. The material that is truly used as an item gives people a material and spiritual enjoyment. The objective characteristics of Ceramic materials are the basis of subjective characteristics, while the subjective characteristics are the sublimation of objective characteristics. The two complement each other and their roles in design are also different [23]. The difference in Ceramic materials gives people different visual and tactile feelings. People's understanding of Ceramic materials relies mostly on different angles of light. Subjective characteristics are one of the important ways to increase the added value of products. The subjective characteristics 
of material texture, emotion, artistic expression and so on must be compatible with the established humanistic connotation and artistic style of the product, so as to satisfy people's status and aesthetic taste. Or spiritual needs such as value orientation.

The color difference and mechanical properties of each group were compared before and after the weather resistance test, soil corrosivity test and damp heat oxygen stability test. Use resources to eliminate all inventory and waste. Achieve the best value for customers with the least amount of investment. In production, the dielectric constant can be flexibly adjusted according to actual needs and can also be used in the preparation of multilayer chip components. To a large extent, problems such as deformation and damage of components due to large differences in sintering shrinkage curves or thermal expansion coefficients between different system Ceramic materials are avoided. The slope of the regression line is closer to the square of the correlation coefficient, and the regression line intercept is closer, so the prediction result is better. As shown in Table 1 and Fig. 2.

Table 1 Comparison of model prediction capabilities

\begin{tabular}{|c|c|c|}
\hline Model & Regression Line & Squared correlation coefficient \\
\hline Hybrid model algorithms & 6.67 & 7.25 \\
\hline Recursive function & 7.95 & 11.62 \\
\hline
\end{tabular}

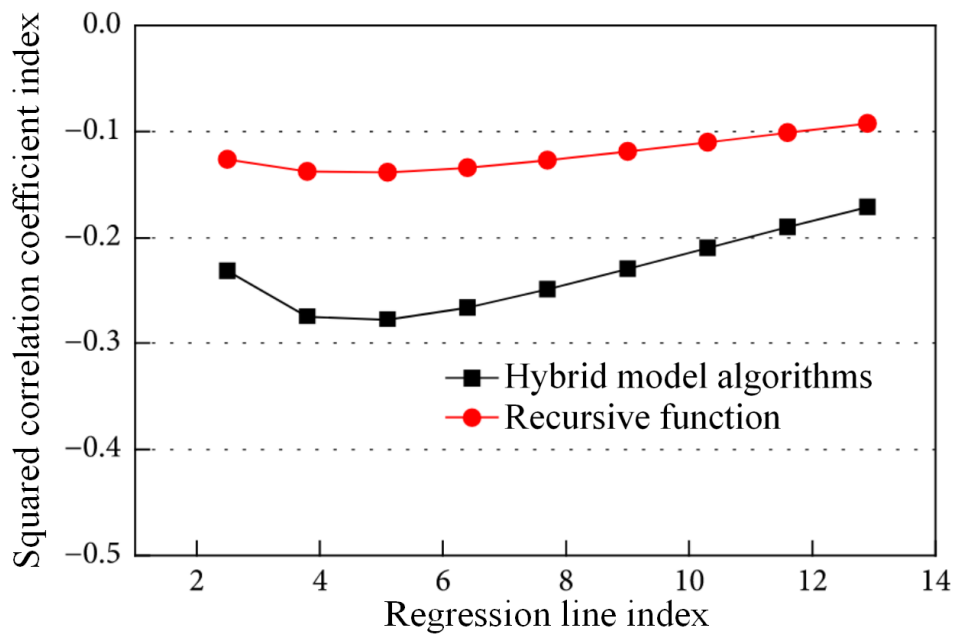

Fig. 2 Comparison of model prediction capabilities

The data of the crystal structure can be obtained by the crystal structure refining method, and the phase content can also be quantitatively analyzed. The formula for quantitative analysis of the phase by the crystal structure refinement method is as follows:

$$
\begin{aligned}
& E_{L R}^{i j}=\sqrt{\sum_{u=1}^{U} \phi_{u}^{i j}}, j \in(1, M), i \in(1, H) \\
& \phi_{u}^{i j}=\left(S_{L u}^{i}-S_{R u}^{i}\right)^{2}
\end{aligned}
$$

The density of the sample was tested using a Swiss METTLER analytical balance. The test principle of the instrument is the drainage method, which is calculated as follows:

$$
w_{j}^{i}=\frac{\frac{1}{\left(E P_{L R}^{i j}\right)^{2}}}{\sum_{j=1}^{k} \frac{1}{\left(E P_{L R}^{i j}\right)^{2}}}, j \in(1, k)
$$

In the open cavity resonance method, when the dielectric resonator operates in the vibration mode, the following characteristic equation is satisfied:

$$
\left(x_{L T}^{i}, y_{L T}^{i}\right)=\sum_{j=1}^{k} w_{j}^{i}\left(x_{R T_{j}}^{i}, y_{R T_{j}}^{i}\right)
$$

The Ceramic materials and the texture they express are also worthy of consideration and research in the field of product design. If the Ceramic materials and their texture can be scientifically and rationally applied, the products that are distributed to the consumers will be more human. China has experienced a long period of material scarcity, and has formed mindsets and applications that focus on the objective characteristics of applied Ceramic materials in product design. However, this old concept and program can no longer meet the requirements of current social development, and can not meet the growing diversified consumer demand of the public. The state has relevant policies and regulations on the design and manufacture of some products. Therefore, the choice of Ceramic materials must meet the requirements of national policies and regulations. Product design can not stay at the objective characteristic stage of the applied Ceramic materials, but should shift the focus to the exploration and application of the subjective characteristics of the Ceramic materials, in order to create more diversified product values, thus adapting to the diverse needs of consumers. Although socio-cultural factors are not a decisive factor in the choice of Ceramic materials, they also play an important role in whether products made from Ceramic materials can be accepted in society. 


\section{Innovative Application of Subjective Characteristics of Ceramic materials}

\subsection{The General Application Range of Breakthrough Ceramic materials}

The exploration and application of the subjective characteristics of Ceramic materials can start from the aspects of application scope, modeling characteristics, composite texture and personality temperament, breaking the inherent program and innovative application methods. For a long time, industrial design has created a modern lifestyle and living environment for human beings, and has also accelerated the consumption of resources and energy, and caused tremendous damage to the ecological balance of the earth. As an objective substance, Ceramic materials do not have any emotional awareness. Their subjective characteristics are partly due to their long-term application in certain products [24]. The over-commercialization of industrial design has made design an important medium to encourage uncontrolled consumption. The planned abolition of goods is the extreme manifestation of this phenomenon, which has led to many criticisms and blame, forcing designers to rethink the role and role of industrial design. And if you consciously broaden the scope of application of Ceramic materials, let people touch them at different times or scenes, you can gradually change the public's subjective feelings.

The non-harmonic effect of the vibration of the composite material can be simply described as a monophonic absorption peak accompanied by a fixed damping coefficient resulting in the existence of dielectric loss. The comparison between the learned value of the flexural strength and the experimental value is shown in Table 2 and Fig. 3.

Table 2 Experimental and predicted values of material flexural strength

\begin{tabular}{|c|c|c|}
\hline Group No & Experimental value & Predictive value \\
\hline 1 & 67.41 & 66.54 \\
\hline 2 & 68.58 & 59.26 \\
\hline 3 & 52.78 & 53.57 \\
\hline 4 & 55.33 & 55.48 \\
\hline
\end{tabular}

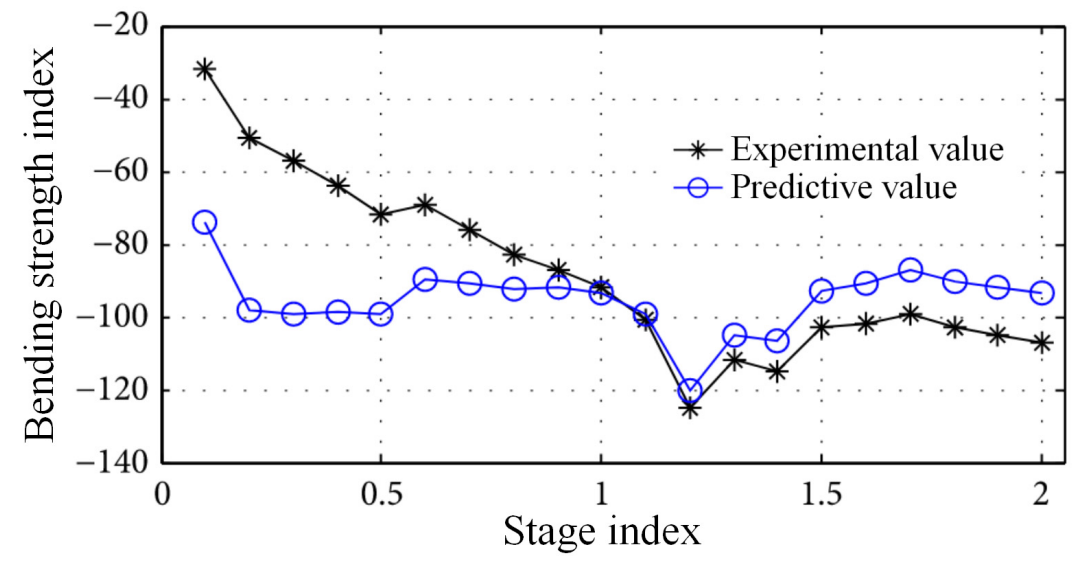

Fig. 3 Comparison of experimental values and predicted values of material flexural strength

When the heat capacity of the heat exchange fluid is small, especially for air, the temperature difference between the inlet and the outlet of the heat exchange fluid is large, and the temperature drop of the heat exchange fluid will inevitably affect the phase change heat storage process:

$$
H W t=\frac{\sum_{i=1}^{N} D_{i}(x)}{N}
$$

For composite systems:

$$
D_{i}=a+\sum_{j=1}^{n} b_{j} p_{j}+r_{i} Y+u
$$

The melt is in a supercooled state when the melt temperature is below the melting point of the composite. The difference between the melt temperature and the melting point of the composite is the degree of subcooling:

$$
D_{i}=a+\sum_{j=1}^{n} b_{j} \ln \left(p_{j}\right)+r_{i} \ln (Y)+u
$$

The phase change driving force of the system is:

$$
\ln \left(D_{i}\right)=a+\sum_{j=1}^{n} b_{j} \ln \left(p_{j}\right)+r_{i} \ln (Y)+u
$$

The high-voltage electric pulse charging and discharging device based on functional Ceramic materials can advance the design problems and quality problems that may occur in the project through collision detection and the like. The performance parameters before and after optimization are shown in Table 3. The topology reliability optimization simulation is compared as shown in Fig. 4. 
Table 3 Performance parameters of the topology before and after optimization

\begin{tabular}{|c|c|c|}
\hline & Before optimization & Optimized \\
\hline Rows & 36 & 58 \\
\hline Number of columns & 22 & 30 \\
\hline Monitoring points & 792 & 1740 \\
\hline
\end{tabular}

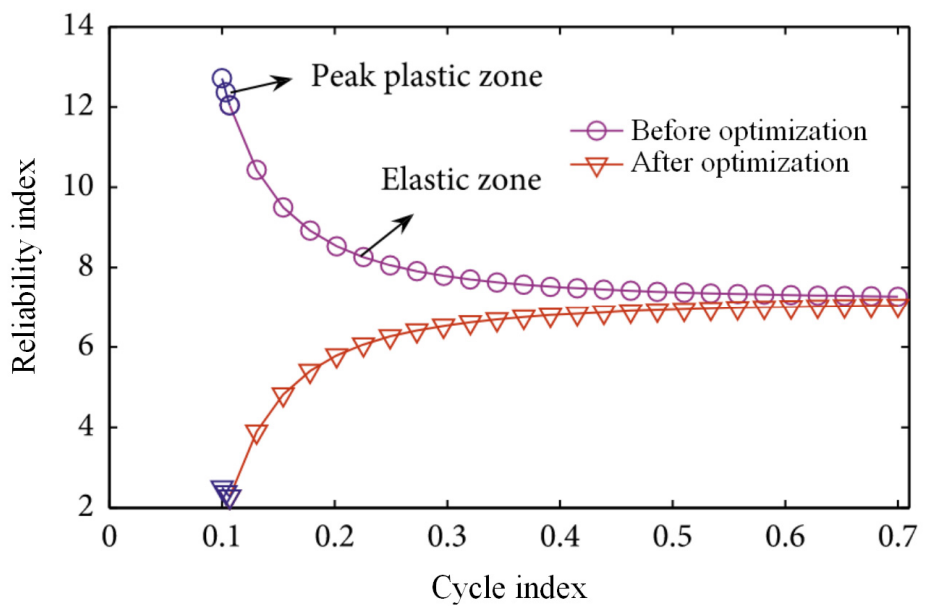

Fig. 4 Simulation comparison of optimized topology reliability of pulse charging and discharging device

The dark indication of material texture is a sentimental expression. The psychological, social or cultural symbolic value of the product in the use environment, that is, the personal association and social culture. Ceramic materials have their own individual temperament, they can express their own beauty through their own language through the inner spirit, and one of the essentials of design is to transform the individuality of the material into a familiar expression of the public through appropriate channels. The innovative application of Ceramic materials has its own advantages. It should be applied comprehensively in design practice. At the same time, it must also strengthen the subjective characteristics of Ceramic materials by combining factors such as form, color, ornamentation and packaging, and fully reflect the inherent temperament of the products. With the development and progress of society, the great enrichment of Ceramic materials, and the further elaboration of consumption levels, people's demand for spiritual functions of products has continuously increased.

\subsection{Exploration and Innovation of Material Characteristics}

From the building Ceramic materials, it can be clearly felt that the psychological experience brought by different material affinity is different. A street or a square, if it is a large piece of cement, will immediately give people a sense of oppression. After experiencing the style of modern science and technology, the design began to change to the postmodern humanistic taste style, and the aesthetic standard of the material changed accordingly [25]. In addition to expressing its functional purpose, product modeling also conveys the cultural connotation of the product through its semantic features, reflecting the contemporary sense and value orientation of a particular society [26]. The packaging of the products adopts recycled paper Ceramic materials, which echoes the main body of the products through the material texture and decorative style of the packaging, which strengthens the subjective characteristics of the bamboo to a certain extent and further highlights the humanistic temperament of the products. In product design, the application of material sensation characteristics should reflect the satisfaction of people's sense of touch and vision, and thus cause emotional pleasure.

In the product design, the original sensory characteristics of the material should be reasonably applied, and the material's realism and simplicity and subtle natural feeling should be fully expressed. In order to achieve positive and negative pulse charging of the lithium ion battery, the same circuit can be used to discharge the lithium ion battery. As a power conversion method commonly used in modern high-voltage electric pulse discharge devices, the basic working principle of pulse charge and discharge is to control the on and off of power devices. When the instruction vector acts on other sectors, the order of action of each basic vector can be obtained. The summary is shown in Table 4.

Table 4 Switch vector assignment in each sector

\begin{tabular}{|c|c|c|c|c|c|c|}
\hline Sector & \multicolumn{5}{|c|}{ Amount of action vectors arranged in chronological order } \\
\hline 1 & 031 & 020 & 120 & 131 & 201 & 023 \\
\hline 2 & 021 & 010 & 103 & 130 & 022 & 203 \\
\hline 3 & 021 & 021 & 023 & 032 & 021 & 401 \\
\hline 4 & 021 & 102 & 013 & 022 & 121 & 203 \\
\hline
\end{tabular}


Under the same phase change driving force, the critical nucleus radius of the two should be the same for the spherical nucleus generated in the free space and the nucleus generated on the foreign base plane. The shape success of the critical nucleus should be:

$$
\mathrm{o}_{\mathrm{j}}(t)=f\left(\left[\sum_{i=1}^{n} w_{i j} x_{i}\left(t-\tau_{i j}\right)\right]-T_{i j}\right)
$$

If the fluid phase is a melt, the rate of nucleation on the plane of the substrate can be expressed by:

$$
\min \Phi(\omega, \xi)=\frac{1}{2}\|\omega\|^{2}+C \sum_{i=1}^{N} \xi_{i}
$$

The effect of the stirring solution on the growth rate of the composite can be expressed by the following formula:

$$
\left.Q\left(u_{i j}\right)=\sum_{i=1}^{n} \operatorname{Maj}_{1 \leq j \leq m} \underset{x}{a x}(T)\right\}
$$

The texture of the material is visual and tactile, as well as psychological association and symbolism through the surface features of the product. In product design, only by fully understanding and understanding the sensory characteristics of the material can it be used reasonably in the design. The perfect shape, color and function are important, and the material texture can not be ignored, it will affect the final effect of product design. Realizing the practical function of the product is the value of product design, and satisfying the psychological aesthetic needs of people is also the product design that should be possessed. The application of material sensation characteristics in product design should focus on the inherent expressiveness of Ceramic materials and the expression of the natural texture of Ceramic materials. On the other hand, it is necessary to actively explore new techniques for material processing and surface decoration, expand the application of Ceramic materials for human texture, and enrich the texture expression of products. The experimental and predicted values of material resistivity are shown in Table 5 and Fig. 5.

Table 5 Experimental and predicted values of material resistivity

\begin{tabular}{|c|c|c|}
\hline Group No & Experimental value & Predictive value \\
\hline 1 & 186.32 & 185.47 \\
\hline 2 & 196.86 & 184.82 \\
\hline 3 & 231.59 & 231.98 \\
\hline 4 & 199.34 & 198.74 \\
\hline 5 & 217.69 & 217.67 \\
\hline
\end{tabular}

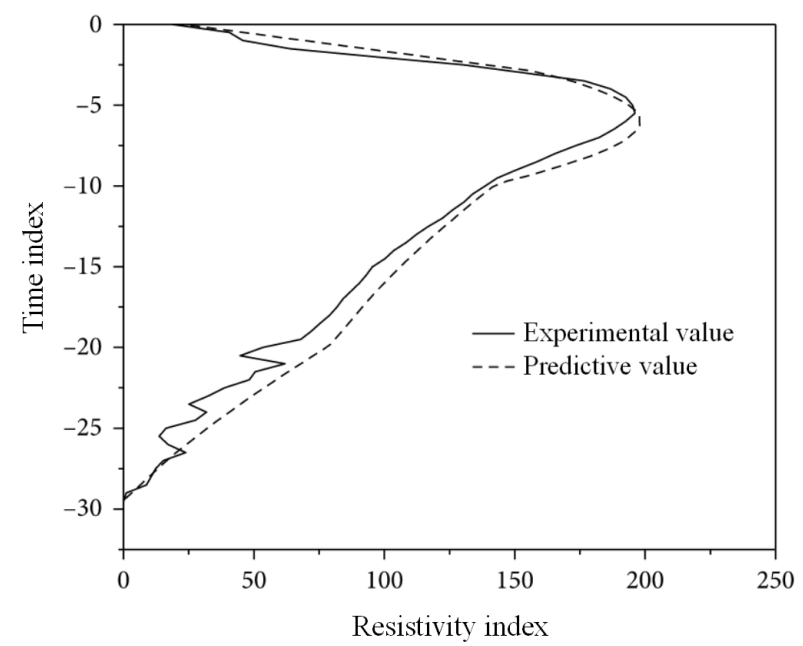

Fig. 5 Comparison of experimental values of material resistivity with predicted values

A series of pulse sequences of equal amplitude and unequal width are output, and the width of the pulse is modulated according to a certain rule. You can change the output voltage or change the output frequency. Thereby greatly improving the dynamic response of the system. Calculate the comparison:

$$
R S R P_{n_{i}, n}+\left(\lambda_{j}-\lambda_{R}\right)=R S R P_{n_{i}, m}
$$

Number of turns of the low voltage side isolation transformer winding:

$$
A^{T} G=\sum_{i} A_{i}^{T} G_{i}
$$

Finally, calculate the number of turns of the winding on the high-voltage side isolation transformer:

$$
S^{*}=\arg \{f(S)\}=\sum_{i=1}^{n} \max \left\{\left(T_{s_{m, j}, m}-D_{s_{m, j}}\right), 0\right\} \rightarrow \min
$$

Since the material sensibility is a comprehensive impression of the material, the visual and tactile texture of the material is scientifically expressed according to the characteristics of the product, thereby giving people a pleasant physiological and psychological feeling. The relationship between the material and its inherent styling characteristics should be consciously eliminated. Without prejudice to the overall design requirements of the product, the brand new styling features will bring consumers a new psychological experience, thus forming a new subjective 
feature. Product designers should be familiar with the performance characteristics of different Ceramic materials, in-depth analysis and research on the semantics of material texture, increase the emotional components by selecting appropriate Ceramic materials, enhance the affinity between products and people, and make products interact with people. Stronger. In production, the dielectric constant can be flexibly adjusted according to actual needs, and can also be used in the preparation of multilayer chip components. To a large extent, problems such as deformation and damage of components due to large differences in sintering shrinkage curves or thermal expansion coefficients between different system Ceramic materials are avoided. Compare the hybrid model algorithm with the recursive function model. The slope of the regression line is closer to the square of the correlation coefficient, and the regression line intercept is closer, so the prediction result is better. As shown in Table 6 and Fig. 6.

Table 6 Comparison of model prediction capabilities

\begin{tabular}{|c|c|c|}
\hline Model & Regression Line & Squared correlation coefficient \\
\hline Hybrid model algorithms & 5.68 & 6.26 \\
\hline Recursive function & 8.96 & 13.63 \\
\hline
\end{tabular}

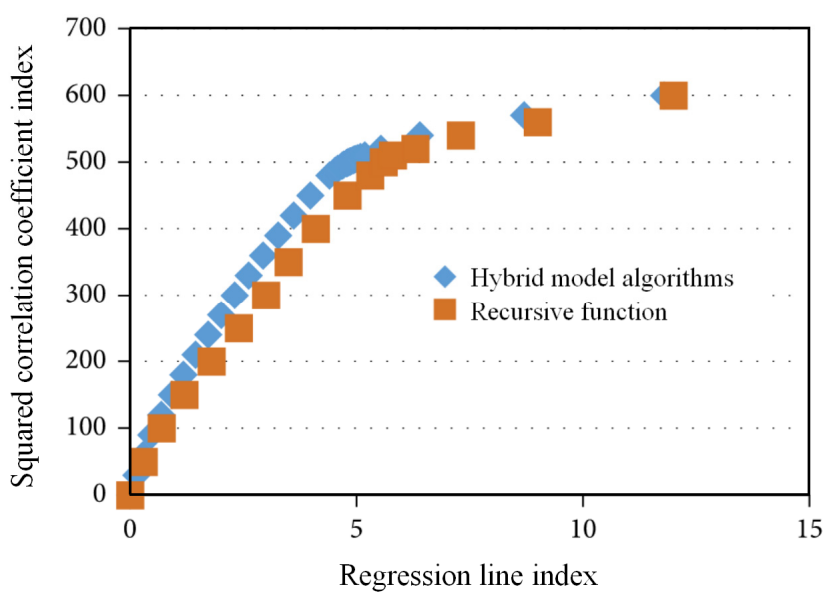

Fig. 6 Comparison of model prediction capabilities

The composite material structure refinement method is used to obtain the data of the composite structure, and the phase content can also be quantitatively analyzed. The formula for quantitative analysis of the phase using the composite structure refinement method is as follows:

$$
E S_{i}=\sum_{j}\left(1-\sum_{q} p_{i q} m_{j q}\right), q \neq i, j
$$

In the open cavity resonance method, when the dielectric resonator operates in the vibration mode, the following characteristic equation is satisfied:

$$
A E_{i}=E S_{i} / S_{i}=\sum_{j}\left(1-\sum_{q} p_{i q} m_{j q}\right) / \sum_{j}
$$

The rational use of the material's sensory characteristics can improve the overall amenity of the product and make people feel the pleasure of use. The product is ultimately used by the user, so the material should be protected during production and use to ensure the health and health of the body and property from injury or loss. Because modern people's pursuit of nature and nature in the high-tech era is more intense, people's psychological aesthetic tendency is more concerned with the natural and authentic nature of natural texture. In the product design, when the physical and sensory properties of the material are triggered as the inner meaning of the product, they will be more closely integrated with the design theme and content, making the product more vivid and more intense artistic charm. In the product design, making full use of the sensory characteristics of the material plays a very good role in giving full play to the function of the product and improving the applicability of the product. With the development of new Ceramic materials and the development of surface treatment technology, the texture effect of Ceramic materials will become more colorful, so we must actively expand the application of material artificial texture to meet the psychological needs of people seeking new and seeking.

\section{Summary}

The material has both primary and objective dual design characteristics. The objective characteristics provide material security for the realization of product functions, while the subjective characteristics are an effective way to create personalized added value. Excellent design is inseparable from beautiful Ceramic materials, but this does not mean that the beauty of the material can override other design elements. The texture of the material is closely related to the product. In addition to giving the product a better function, the material with a certain texture will affect the overall style of the product. With the increasing awareness of environmental protection, the choice of Ceramic materials in product design is no longer just to meet the requirements and functions of the products, but more attention should be paid to the compatibility of Ceramic materials in the process of extraction, preparation, 
production and use and disposal of products. Sex and coordination. Reasonable texture design should not only meet the functional needs of the product, but also meet the psychological needs of people, and in line with the principle of sustainable development of society. In product design, it is necessary to fully understand the sensory characteristics of Ceramic materials, timely grasp the development trend of new technologies, new processes and new Ceramic materials, and use appropriate methods to deal with appropriate Ceramic materials to maximize the respective characteristics of the Ceramic materials. Thereby achieving the perfect expression of the product texture, bringing a comprehensive feeling of natural, rich and intimate physiological senses, and enabling people to obtain emotional satisfaction.

\section{References}

1. Haug A. Acquiring Materials knowledge in design education[J]. International Journal of Technology and Design Education, 2018(2):1-16.

2. Chang, Hsiang-Tang, Lu, Chien-Han. Simultaneous Evaluations of Material Toxicity and Ease of Disassembly during Electronics Design[J]. Journal of Industrial Ecology, 2014, 18(4):478-490.

3. Miyashita L, Ishihara $\mathrm{K}$, Watanabe $\mathrm{Y}$, et al. ZoeMatrope: a system for physical material design[J]. ACM Transactions on Graphics, 2016, 35(4):1-11.

4. Dupont T, Leclaire P, Panneton R, et al. A microstructure material design for low frequency sound absorption[J]. Applied Acoustics, 2018, 136:86-93.

5. Khare V, Sonkaria S, Lee G Y, et al. From 3D to 4D printing - design, material and fabrication for multi-functional multi-Materials[J]. International Journal of Precision Engineering and Manufacturing-Green Technology, 2017, 4(3):291-299.

6. Reichmanis E, Katz H, Kloc C, et al. Plastic electronic devices: From Materials design to device applications[J]. Bell Labs Technical Journal, 2014, 10(3):87-105.

7. Sakimoto K K, Kornienko N, Yang P. Cyborgian Material Design for Solar Fuel Production: The Emerging Photosynthetic Biohybrid Systems[J]. Accounts of Chemical Research, 2017, 50(3):476-481.

8. Tang Y, Zhang Y, Li W, et al. ChemInform Abstract: Rational Material Design for Ultrafast Rechargeable Lithium-Ion Batteries[J]. Chemical Society Reviews, 2015, 44(17):5926-5940.

9. Banh $\mathrm{T} T$, Lee D. Multi-material topology optimization design for continuum structures with crack patterns $[\mathrm{J}]$. Composite Structures, 2017, 186:193-209.

10. Chen Y, Cheng X, Fu K. Multi-material design of a vehicle body considering crashworthiness safety and social effects[J]. International Journal of Crashworthiness, 2019:1-10.
11. Laura Remón, Siedlecki D, Cabeza-Gil I, et al. Influence of material and haptic design on the mechanical stability of intraocular lenses by means of finite-element modeling $[\mathrm{J}]$. Journal of Biomedical Optics, 2018, 23(3):1-10.

12. Liliana C. Tomé, Marrucho I M. Ionic liquid-based Materials: A platform to design engineered $\mathrm{CO} 2$ separation membranes[J]. Chemical Society Reviews, 2016, 45(10):2785-2824.

13. Onishi T. Quantum Chemistry in Proton-Conductors. Mechanism Elucidation and Materials Design[J]. Advances in Quantum Chemistry, 2015, 70:31-67.

14. Grujicic M, Snipes J S, Ramaswami S. Application of the Materials-by-Design Methodology to Redesign a New Grade of the High-Strength Low-Alloy Class of Steels with Improved Mechanical Properties and Processability[J]. Journal of Materials Engineering and Performance, 2015,

15. Heath A C G, Kwak M L. Ensuring the nomenclatural stability of Ixodes anatis Chilton, 1904 with the discovery of lost type material and the designation of a lectotype[J]. New Zealand Entomologist, 2019(4):1-2.

16. Li W, Shi H, Zhang J. From Molecules to Materials: Computational Design of N-Containing Porous Aromatic Frameworks for $\mathrm{CO} 2$ Capture[J]. ChemPhysChem, 2014, 15(9):1772-1778.

17. Souto N, Andrade-Campos A, Thuillier S. Mechanical design of a heterogeneous test for material parameters identification[J]. International Journal of Material Forming, 2016, 10(3):353-367.

18. Wang F, Grinberg I, Jiang L, et al. Materials Design of Visible-Light Ferroelectric Photovoltaics from First Principles[J]. Ferroelectrics, 2015, 483(1):1-12.

19. Desai S, Dean C. Concurrent material and process selection in a flexible design for manufacture paradigm $[\mathrm{J}]$. International Journal of Advanced Manufacturing Technology, 2018, 97(8):764-769.

20. Al-Bahi A M, Soliman A Y A, Hassan M H M, et al. Concept design of an illicit material detection system[J]. Journal of Radioanalytical and Nuclear Chemistry, 2014, 299(1):351-356.

21. Gorley M, Fursdon M, Kalsey M. Integrating Materials Engineering and Design for Fusion[J]. IEEE Transactions on Plasma Science, 2018, PP(99):1-6.

22. Demis S, Tapali J G, Papadakis V G. Plant Design and Economics of Rice Husk Ash Exploitation as a Pozzolanic Material[J]. Waste and Biomass Valorization, 2015, 6(5):843-853.

23. Travitzky N, Bonet A, Dermeik B, et al. Additive Manufacturing of Ceramic-Based Materials[J]. Advanced Engineering Materials, 2014, 16(6):729-754.

24. Tiina Härkäsalmi, Lehmonen J, Jukka Itälä, et al. Design-driven integrated development of technical and perceptual qualities in foam-formed cellulose 
fibre Materials[J]. Cellulose, 2017, 24(11): 5053-5068.

25. Hamim S U, Singh R P. Taguchi-based Design of Experiments in Training POD-RBF Surrogate Model for Inverse Material Modeling using Nanoindentation[J]. Inverse Problems in Science and Engineering, 2016, 25(3):363-381.

26. Elhebeary M, Saif M T A. Design, Simulation, and Testing of a Novel Bending Stage for Mechanical Characterization of Materials[J]. Experimental Mechanics, 2016, 57(1):1-8. 